\title{
Distinct deposition of amyloid- $\beta$ species in brains with Alzheimer's disease pathology visualized with MALDI imaging mass spectrometry
}

\author{
Nobuto Kakuda ${ }^{1+}$, Tomohiro Miyasaka ${ }^{2+}$, Noriyuki Iwasaki ${ }^{3}$, Takashi Nirasawa ${ }^{3}$, Satoko Wada-Kakuda ${ }^{2}$, \\ Junko Takahashi-Fujigasaki ${ }^{4}$, Shigeo Murayama ${ }^{4}$, Yasuo Ihara ${ }^{5}$ and Masaya Ikegawa ${ }^{1 *}$
}

\begin{abstract}
Amyloid $\beta(A \beta)$ deposition in the brain is an early and invariable feature of Alzheimer's disease (AD). The A peptides are composed of about 40 amino acids and are generated from amyloid precursor proteins (APP), by $\beta$ - and $\gamma$-secretases. The distribution of individual $A \beta$ peptides in the brains of aged people, and those suffering from $A D$ and cerebral amyloid angiopathy (CAA), is not fully characterized. We employed the matrix-assisted laser desorption/ ionization-imaging mass spectrometry (MALDI-IMS) to illustrate the spatial distribution of a broad range of A species in human autopsied brains. With technical advancements such as formic acid pretreatment of frozen autopsied brain samples, we have: i) demonstrated that $A \beta 1-42$ and $A \beta 1-43$ were selectively deposited in senile plaques while full-length $A \beta$ peptides such as $A \beta 1-36,1-37,1-38,1-39,1-40$, and $A \beta 1-41$ were deposited in leptomeningeal blood vessels. ii) Visualized distinct depositions of $\mathrm{N}$-terminal truncated $A \beta 40$ and $A \beta 42$, including pyroglutamate modified at Glu-3 (N3pE), only with IMS for the first time. iii) Demonstrated that one single amino acid alteration at the C-terminus between $A \beta 1-42$ and $A \beta 1-41$ results in profound changes in their distribution pattern. In vitro, this can be attributed to the difference in the self-aggregation ability amongst $A \beta 1-40, A \beta 1-41$, and $A \beta 1-42$. These observations were further confirmed with immunohistochemistry $(\mathrm{IHC})$, using the newly developed anti-A $1-41$ antibody. Here, distinct depositions of truncated and/or modified C- and N-terminal fragments of ABs in AD and CAA brains with MALDI-IMS were visualized in a spacio-temporal specific manner. Specifically, AB1-41 was detected both with MALDI-IMS and IHC suggesting that a single amino acid alteration at the $C$-terminus of $A \beta$ results in drastic distribution changes. These results suggest that MALDI-IMS could be used as a standard approach in combination with clinical, genetic, and pathological observations in understanding the pathology of AD and CAA.
\end{abstract}

Keywords: Amyloid $\beta$, Alzheimer's disease, Cerebral amyloid angiopathy, Imaging mass spectrometry, C- and $\mathrm{N}$-terminal variations of $\mathrm{A} \beta$, Senile plaques, $\gamma$-secretase, Perivascular space

\section{Introduction}

Precise molecular identification of pathological depositions accelerates the diagnosis and clarifies the pathogenesis of neurodegenerative disorders [10]. In Alzheimer's disease $(\mathrm{AD})$ brains, depositions of insoluble amyloid $\beta(\mathrm{A} \beta)$ are

\footnotetext{
* Correspondence: mikegawa@mail.doshisha.ac.jp

${ }^{\dagger}$ Equal contributors

${ }^{1}$ Genomics, Proteomics and Biomedical Functions, Department of Life and Medical Systems, Faculty of Life and Medical Sciences, Doshisha University, Kyoto, Japan

Full list of author information is available at the end of the article
}

detected in senile plaques (SP) before disease onset [1-3, $22,23]$. In addition to SP in the brain, $A \beta$ is also deposited in the walls of cerebral capillaries and arteries and causes cerebral amyloid angiopathy (CAA) [27, 30, 32]. Although $A \beta 1-42$ is predominant in $S P$, other $A \beta$ variants, including $\mathrm{N}$-terminal or $\mathrm{C}$-terminal truncated or modified $A \beta s$, are also identified in affected AD brains $[4,6,18]$. Characterizing and visualizing the broad $A \beta$ species is needed to understand the $\mathrm{A} \beta$-production, -metabolism, and -deposition, and may help elucidate the pathogenesis of $\mathrm{AD}$ and CAA. 
In classical AD neuropathology, immunohistochemistry has been used to determine the localization of $\mathrm{A} \beta \mathrm{s}$ in brain tissues. However, the reliability of the results highly depends on the performance of antibodies, and the method cannot distinguish different variants when several epitopes are used simultaneously. Therefore, unbiased mass spectrometry-based proteomic analysis is a valuable approach to characterize the variety of $A \beta$ species in brain tissues $[9,25,26]$. The matrix-assisted laser desorption/ionization (MALDI) mass spectrometric method has revealed different $A \beta$ isoforms in homogenized brain lysates with immuno-precipitated samples [15]. However, this approach failed to reveal the detailed distribution of $A \beta$ in the brain, and again, is dependent on the affinity of antibodies used. In recent years, MALDI-imaging mass spectrometry (IMS) has emerged as a powerful tool for investigating the distribution of proteins and small molecules within biological systems, through the in-situ analysis of tissue sections [21]. Here, we adopt this technology on postmortem brain tissues to extend and create a comprehensive protein mapping.

To analyze human brain samples, a novel protocol using formic acid pretreatment of brain tissues and an advanced type mass spectrometry, that bears advantages in its rapidity, sensitivity, and reproducibility, was needed. With the current technical advancements, we have successfully visualized distinct depositions of $\mathrm{N}$ and $\mathrm{C}$-terminal variations of $\mathrm{A} \beta$ in pathological human autopsied brains. The current strategy provides new insights into understanding the neuropathology of AD and $\mathrm{CAA}$ in terms of $\mathrm{A} \beta$ metabolism.

\section{Materials and methods}

\section{Patients and brain specimens}

Human cortical specimens for IMS and IHC were obtained from the Brain Bank at Tokyo Metropolitan Institute of Gerontology. Brains were removed, processed, and stored at $-80{ }^{\circ} \mathrm{C}$ within $8 \mathrm{~h}$ postmortem. Patients were placed in a cold $\left(4{ }^{\circ} \mathrm{C}\right)$ room within $2 \mathrm{~h}$ after death. All brains registered at the brain bank came with written informed consents for their use in medical research from the patients or their families. Each brain specimen was taken from the occipital cortex of five AD patients and five age-matched controls. This study was approved by the ethics committee at Doshisha University and Tokyo Metropolitan Geriatric Hospital.

In the present study, the extent of $A \beta$ deposition as shown by an $\mathrm{A} \beta$ monoclonal antibody (12B2, 1:50 dilution; IBL, Gunma, Japan), was defined by Braak SP (amyloid) stages [2,3]. At stage $\mathrm{O}$, there are almost no senile plaques throughout the isocortex. At stage A, low densities of $\mathrm{A} \beta$ deposits are detected in the isocortex, particularly in the basal portions of the frontal, temporal, and occipital lobes. Furthermore, some plaques are found in the presubiculum and Pre- $\beta$ and Pre- $\gamma$ layers of the entorhinal complex. Stage B shows an increase in $A \beta$ deposits in almost all isocortical association areas, and only the primary sensory areas and primary motor field remain practically devoid of deposits. There are mild amounts of deposits in the hippocampal formation, and $A \beta$ deposits may be found in the entorhinal cortex. At stage $C$, virtually all the isocortical areas are affected, whereas deposits in the hippocampal formation display the same pattern as that of stage B. AD brains are invariable at stage $C$.

\section{Imaging mass spectrometry}

Frozen tissue sections were cut on a cryostat (CM1950, Leica Microsystems, Wetzlar, Germany) at a $10 \mu \mathrm{m}$ thickness using indium-tin-oxide-coated glass slides (Bruker Daltonics, Bremen, Germany). Prior to washing, stored samples were placed in a vacuum chamber to dry. To remove endogenous lipids and inorganic salts, dried samples were immersed in $70 \%$ ethanol for $30 \mathrm{~s}$, pure ethanol for $30 \mathrm{~s}$, Carnoy's solution for $3 \mathrm{~min}$, pure ethanol for $30 \mathrm{~s}$, $0.1 \%$ TFA for $1 \mathrm{~min}$, and pure ethanol for $30 \mathrm{~s}$. Prior to matrix coating, treated with a formic acid vapor. Sinapinic acid was used as a matrix. For mass spectrometric measurements, tissue areas were defined using the FlexControl 3.8 and FlexImaging 5.0 software packages (both Bruker Daltonics). Spectra were acquired using the rapifleX MALDI Tissuetyper (Bruker Daltonics) in positive linear mode, where ions were detected in a mass range of $\mathrm{m} / \mathrm{z}$ 2000 to 20,000, with spatial resolution of 20 and $100 \mu \mathrm{m}$, respectively. A ready-made protein standard was used for spectra calibration (Bruker Daltonics). Visualization and statistical analysis were completed using FlexImaging and SciLS Lab 2016a (SCiLS, Bremen, Germany).

\section{Immunohistochemistry}

Fresh frozen sections were post-fixed using 4\% paraformaldehyde (PFA) in phosphate buffered saline (PBS). After a brief wash in PBS, sections were stained by Sudan Black $\mathrm{B}$ for elimination of the autofluorescence due to lipofuscin [12]. Following immersion in $10 \%$ goat serum in PBS and $0.1 \%$ Tween20 (PBS-T) for $60 \mathrm{~min}$, the sections were incubated with primary antibodies diluted in 1\% BSA-PBS-T for $18 \mathrm{~h}$ at room temperature. Sections were then rinsed with PBS-T, and bound antibodies were visualized with secondary antibodies conjugated with Alexa dyes (Life Technology). The specimens were visualized by confocallaser-scanning-microscope (LSM 700; Carl Zeiss Inc.). Whenever necessary, bound antibodies were labeled by biotinylated anti-mouse or anti-rabbit IgG antibodies (Vector Laboratories, Inc., Burlingame, CA), followed by avidin and biotinylated HRP complex (Vectastain Elite ABC kit; Vector Laboratories, Inc.). Bound HRP was developed with 3,3-diaminobenzidine (DAB) in the presence of hydrogen peroxide. 


\section{Developing and characterizing anti-A $\beta 41$ polyclonal antibody}

The antigen peptide corresponding to A $\beta 37-41$ amino acid residues, GGVVI, conjugated to thyroglobulin was immunized into rabbits four times at 2-week intervals. Subsequently, the serum was collected and purified with $A \beta 41$ affinity chromatography. To confirm the purified antibodies' specificity, synthetic A $\beta 1-40, A \beta 1-41$, and A $\beta 1-42$ were run on a $12 \%$ Tris-tricine gel $[7,8]$ followed by western blotting. All A $\beta$ s were detected with 82E1 (IBL, Japan), while A $\beta 41$ was detected with purified polyclonal antibody.

\section{$A \beta$ aggregation test with Thioflavin T assay}

$\mathrm{A} \beta$ was dissolved in hexafluoro isopropanol (HFIP) to $1.8 \mathrm{mg} / \mathrm{ml}$. Each of the $230 \mu \mathrm{M} \mathrm{A} \beta$ samples were completely dried up. Samples were then dissolved in $50 \mathrm{mM}$ $\mathrm{NaPO}_{4}$ (pH 7.4) and $0.25 \mathrm{mM} \mathrm{NaOH}$. An equal volume of $56 \mathrm{mM}$ thioflavin $\mathrm{T}$ was then added to each $\mathrm{A} \beta$ sample. Fifty microliters of the mixture was then added into the black wall plate individually and incubated at $37^{\circ} \mathrm{C}$ with preventive light cover. The final $\mathrm{A} \beta$ concentration was $115 \mu \mathrm{M}$ in each well. During sample incubation, thioflavin $\mathrm{T}$ intensity was measured for $24 \mathrm{~h}$, at an excitation wavelength of $465 \mathrm{~nm}$ and emission wavelength of $535 \mathrm{~nm}$.

\section{Results}

\section{Depositions of $A \beta 1-40$ and $A \beta 1-42$ visualized with MALDI-IMS and IHC}

To characterize the broad range of deposited and accumulated $A \beta$ species in postmortem brain tissues, we adopted MALDI-IMS technology combined with formic acid pretreatment of brain tissues. Samples were obtained from sporadic AD patients with CAA $(n=5$; mean age $=83.2$ y) and SP free aged subjects (SP O) brains $(\mathrm{n}=5$; mean age $=77.2 \mathrm{y})$ as shown in Table 1 .

In case No. 4, with the most advanced Braak stage [2, 3], MALDI-IMS analysis identified distinct deposits of A $\beta 1-$ 40 and $A \beta 1-42$ in the $A D$ brain tissue (Fig. 1A, B). The

Table 1 Clinical and pathological data of AD with CAA cases and aged SP O subjects

\begin{tabular}{llllll}
\hline Subject No. & Gender & Age at death & Braak & BraakSP & CAA \\
\hline 1 & M & 83 & 5 & C & 0.5 \\
2 & $M$ & 88 & 5 & C & 1 \\
3 & M & 84 & 5 & C & 2 \\
4 & M & 78 & 6 & C & 1 \\
5 & M & 83 & 5 & C & 1 \\
6 & M & 84 & 1 & 0 & 0 \\
7 & M & 78 & 1 & 0 & 0 \\
8 & $M$ & 70 & 1 & 0 & 0 \\
9 & $M$ & 73 & 1 & 0 & 0 \\
10 & $M$ & 1 & 0 & 0 \\
\hline
\end{tabular}

A $\beta 1-40$ was distributed predominantly in the leptomeningeal vessels of the subarachnoid space and arterioles in the cerebral parenchyma. However, $A \beta 1-42$ formed SP in the cerebral parenchyma, and was mostly deposited in the pyramidal cell layer and subpial molecular layer (Fig. 1A, B). Subpial depositions of A $\beta 1-42$ are seen lining the subarachnoid space, but each MALDI-IMS signal pattern was more dispersed compared with pyramidal layer depositions due to the $100 \mu \mathrm{m}$ pitch resolution. As shown in detail in Fig. $1 C$, the mass spectrum from leptomeningeal blood vessels in the subarachnoid space, arterioles, and cerebral parenchyma displayed different mass numbers corresponding to each $A \beta$ ion. $A \beta 1-40$ was detected not only in the blood vessels, but also along with $A \beta 1-42$ in the cerebral parenchyma (Fig. 1C). The MALDI-IMS signal of $A \beta 1-40$ from CAA is stronger than that of SP in the cerebral parenchyma (Fig. 1A, B). In normal brains, MALDI-IMS detected $A \beta$ distribution as weak dot-like patterns (Additional file 1: Figure S1). Different distributions of $A \beta 40$ and $A \beta 42$ were also demonstrated by IHC using serial frozen tissue sections. The anti-A $\beta 40$ antibody preferentially labeled CAA, which is in clear contrast to the distribution of $A \beta 42$ in the cerebral parenchyma (Fig. 1D). It should be noted that these findings are further confirmed when the bound antibodies were visualized by the avidin-biotin-complexDAB detection method (Additional file 1: Figure S2).

\section{Deposition of full-length $A \beta s$ visualized in human brain with MALDI-IMS}

The CAA phenotypes of case No. 3 were the most advanced amongst subjects of this study. Interestingly, A $1-42$ deposition in the subpial molecular layer was less prominent than in case No. 4 (Fig. 1A, E and Additional file 1: Figure S3). Furthermore, $A \beta 1-36$ to $A \beta 1-41$ were preferentially deposited in the leptomeningeal vascular areas, while both $A \beta 1-42$ and $A \beta 1-43$ were distributed in the cerebral parenchyma of the occipital cortex (Fig. 1E). This is the first study which detected $A \beta 1-41$ in human brain tissue. We have hypothesized that one single amino acid alteration at the $C$-terminus of $A \beta$ results in drastic changes in $A \beta s$ distribution. The spatial resolution used with MALDI-IMS is a key parameter and must be chosen carefully because high-resolution imaging often results in decreased sensitivity. In Fig. 1A and E, MALDI-IMS with $100 \mu \mathrm{m}$ pitch resolution was used, and we obtained an overall distribution profile in a relatively wide area. To portray fine tissue structures, such as vessels, highresolution MALDI imaging $(20 \mu \mathrm{m})$ was performed. The MALDI-IMS clearly demonstrates that A $\beta 1-36$ to A $\beta 1-$ 41 are distributed in the leptomeningeal vessels and arteriole walls and are quite different from the SP distribution of $A \beta 1-42$ and $A \beta 1-43$ (Additional file 1: Figure S4). These results are in line with recent IHC findings citing 


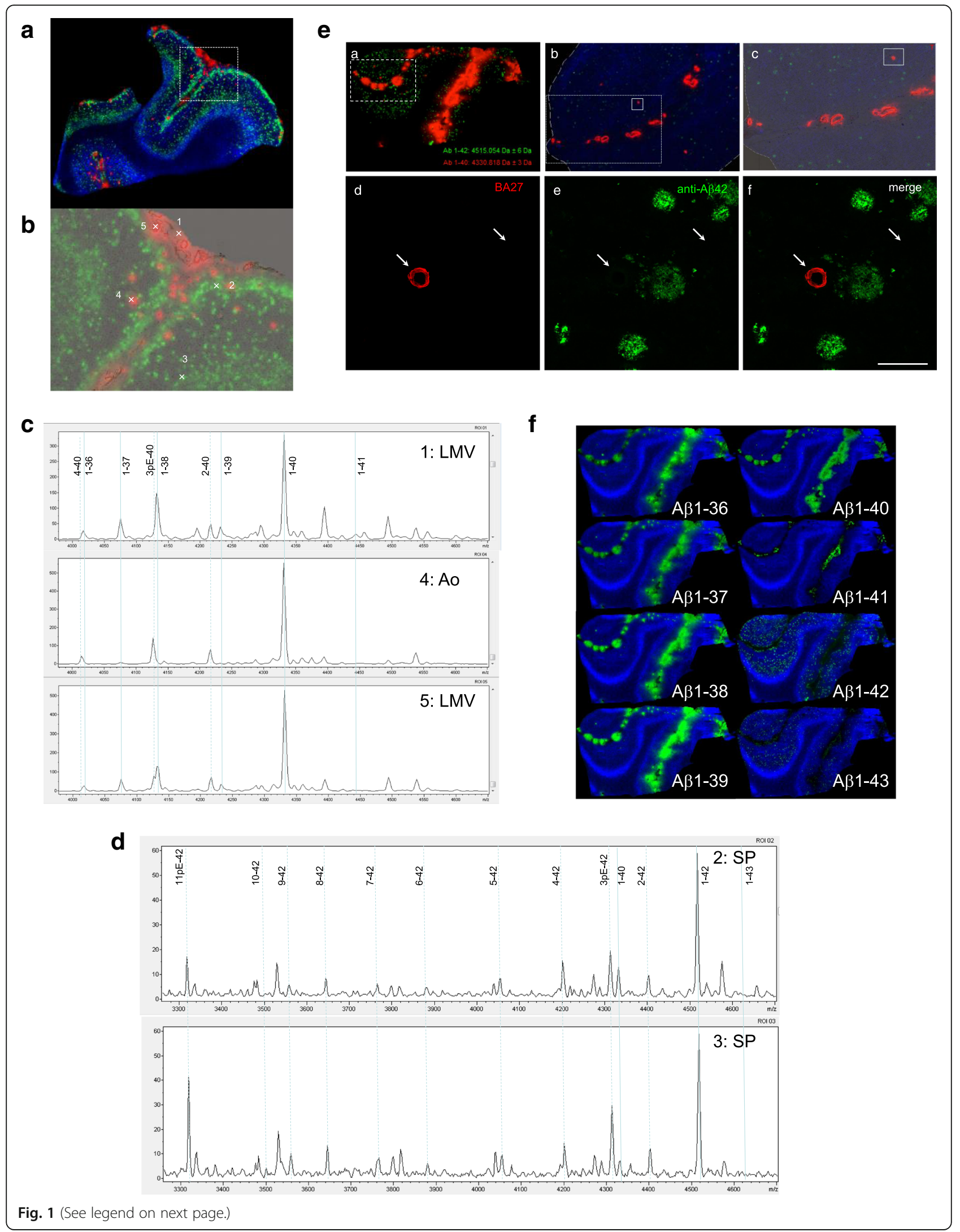


(See figure on previous page.)

Fig. 1 MALDI-IMS for frozen AD brain sections. A: AB1-40 deposits in the leptomeningeal blood vessels and arterioles (red) and AB1-42 deposits in cerebral parenchyma (green). The $\mathrm{m} / \mathrm{z} 4939.9$ was used to detect the tissue structure and shows an unknown biomolecule (blue). B: Optical density for MALDI-IMS. This figure is a magnification of the region within the dotted square in Fig. 1A. A $1-40$ is deposited in leptomeningeal blood vessels ( 1 and 5) and arterioles (4) shown in red. AB1-42 is deposited in cerebral parenchyma as senile plaques ( 2 and 3 ) shown in green. C: MALDI Mass spectrum in leptomeningeal blood vessels (LMV), arterioles (Ao), and senile plaque (SP) of Fig. 1B. A $11-40$ and N-terminal truncated $A \beta x-40$ are located in Ao, while $A \beta 1-36$ to $A \beta 1-41$ are in LMV. $A \beta 1-42, A \beta 1-43$, and N-terminal truncated $A \beta x-42$ are preferentially located in SP. D: MALDI-IMS and IHC of various C-terminal truncated A $\beta$ peptides in AD with severe CAA. (a) MALDI-IMS $100 \mu$ m resolution imaging for A $1-40$ (red) and AB 1-42 (green). (b) Highlight $20 \mu \mathrm{m}$ resolution leptomeningeal blood vessels and cortex imaging in dotted square (a). (c) Highlight of an arteriole in solid square (b). Adjacent sections of the occipital cortex from AD brains were immunostained and focused on arteriole and cerebral parenchyma (c) using antibodies against A 40 (d: BA27) or A 42 (e: anti-A 342 polyclonal) and merged view (f). Both analyses demonstrated that A 40 is preferentially deposited in leptomeningeal blood vessels and arterioles in the subarachnoid space and the cerebral parenchyma forming CAA. In contrast, AB42 is mainly deposited in SP. IHC analysis also demonstrated the differential distribution of A 440 and A 342 , which were CAA dominant and SP dominant deposition, respectively. Solid rectangles indicate the area illustrated in the panel. Scale bars $=100 \mu \mathrm{m}$. E: MALDI-IMS of various C-terminal truncated $A \beta$ peptides in $A D$ with severe CAA (NO. 3). $A \beta 1-36$ to $A \beta 1-41$ are preferentially deposited in leptomeningeal blood vessels, while $A \beta 1-42$ and $A \beta 1-43$ are deposited in the cerebral parenchyma as senile plaques

that not only $A \beta 1-40$, but also $A \beta 37$ to $A \beta 39$, are deposited in the leptomeningeal blood vessel walls [17].

\section{Deposition of $\mathrm{N}$-terminal truncated/modified $\mathrm{A} \beta 40$ and $\mathrm{A} \beta 42$} A variety of different $\mathrm{N}$-truncated $\mathrm{A} \beta$ peptides have been identified starting with amino residue Ala-2, N3pE, Phe-4, Arg-5, Asp-7, Ser-8, Gly-9, Tyr-10, and N11pE [15, 24]. In this study, the mass spectrometric profile of SP and CAA gave rise to serial $A \beta s$ like $N 3 p E-A \beta$ and $N 11 p E-A \beta$ in addition to many $\mathrm{N}$-terminally truncated forms of $\mathrm{A} \beta \mathrm{s}$ (Fig. 1C). As shown here, MALDI-IMS distinguished between $\mathrm{N}$-terminal truncated $\mathrm{A} \beta 40$ and $\mathrm{A} \beta 42$ with individual localization profiles in the brain tissues (Fig. 2). MALDI-IMS demonstrated that N3pE-A 340 is

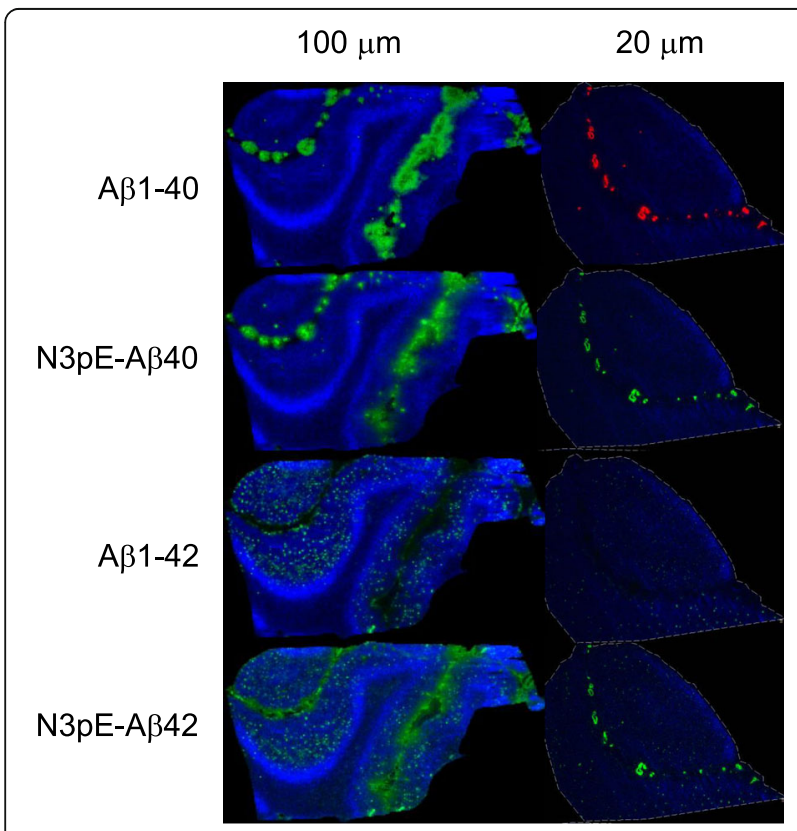

Fig. $2 A \beta 1-40 / 42$ and N3pE-AB40/42 were detected at a $100 \mu m$ and $20 \mu \mathrm{m}$ resolution IMS, respectively. A $1-40$ and N3pE-A 40 preferentially deposited in leptomeningeal blood vessels and arterioles, while A $\beta 1-42$ and N3pE-A 42 deposited in the cerebral parenchyma deposited in the leptomeningeal vessels and arterioles similarly to $A \beta 1-40$, but was not present in SP. The $m /$ $z 4126.5$ for N3pE-A $\beta 40$ and 4132.5 for $A \beta 1-38$ showed close to the mass number respectively (Fig. 1C). However, a detailed analysis with MALDI-IMS demonstrated that the $m / z 4126.5$ for N3pE-A $\beta 40$ was distributed both in leptomeningeal blood vessels and arterioles, while the $\mathrm{m} / z$ 4132.5 for A $1-38$ was detected only in leptomeningeal blood vessels (Fig. 1C). The mass number of N3pE-A $\beta 42$ was detected not only in SP, but also in leptomeningeal blood vessels with MALDI-IMS (Fig. 2 and Additional file 1: Figures S5 and S6). Furthermore, MALDI-IMS obtained the detailed distributions of both $A \beta x-40$ and $A \beta x-42$ $(\mathrm{x}=2, \mathrm{~N} 3 \mathrm{pE}, 4,5,6,7,8,9,10$, and $11 \mathrm{pE})$ in AD accompanied with CAA brains (Additional file 1: Figures S5 and S6). These $N$-truncated $A \beta$ species were largely similar in their distribution to the full-length $A \beta 1-40$ and $A \beta 1-42$. As shown here, MALDI-IMS can individually track the whole distribution of complex molecules having multiple modifications, an advantage over conventional IHC. Not only does C-terminus truncation cause different distribution patterns than $\mathrm{N}$-terminus truncation, (Fig. 1A, B), it is plausible that the structures of its $\mathrm{C}$-terminus structure predefine the dynamics of $A \beta s$ rather than those of the $\mathrm{N}$ terminal end. It must be noted that the N3pE-A $\beta 42$ may particularly behave independently from $A \beta 1-42$. Furthermore, N11pE-A $\beta 42$ was also detected in SP with MALDIIMS as a relatively major peak (Fig. $1 \mathrm{C}$ and Additional file 1: Figures $S 5$ and S6). This pyroglutamate modified $A \beta$ has only been seen in SP [15, 24], while N11pE-A 440 was detected in leptomeningeal vessel walls, but not in SP (Additional file 1: Figures S5 and S6).

Deposition of $A \beta 1-41$ in contrast with $A \beta 1-40$ and $A \beta 1-42$ In Fig. $1 C$ and $E$, we demonstrated the presence of $A \beta 1-$ 41 peptide in leptomeningeal vessels similar to the full length $A \beta$ species, $A \beta 1-36$ to $A \beta 1-40$. However, $A \beta 1-42$ was predominantly deposited within the cerebral parenchyma. A $\beta 1-41$ and $A \beta 1-42$ showed clear differences in 
deposition patterns. To characterize $A \beta 1-41$ in the brain, we have generated antibodies against $A \beta 41$, as shown in Additional file 1: Figure S7. The IHC using anti-A 341 IgG labeled vessels with CAA as $\mathrm{A} \beta 40$, but was not in present in SP and showed a clear contrast to the antibodies against A 342 (Fig. 3A). To further test the hypothesis that such a drastic alteration of $\mathrm{A} \beta$ peptides distribution depends on its self-aggregation ability, we examined the in vitro aggregation characteristics of $A \beta 1-40, A \beta 1-41$, and $A \beta 1-42$ using thioflavin T. Synthetic A $\beta 1-42$ peptide aggregated immediately, while both synthetic $A \beta 1-40$ and $A \beta 1-41$ peptides aggregated slowly during a $24 \mathrm{~h}$ incubation (Fig. 3B). Thus, a C-terminal structure alteration from $A \beta 41$ to $A \beta 42$ is sufficient to alter its self-aggregation ability, and this structural difference may lead to SP formation in the cerebral parenchyma.

\section{$A \beta 38$ and $A \beta 41$ deposit in vessels prior to $A D$ onset}

We analyzed the accumulation of $A \beta 41$ in contrast to $\mathrm{A} \beta 38, \mathrm{~A} \beta 40$, and $\mathrm{A} \beta 42$ in the brain at various stages of
SP. Surprisingly, $A \beta 38$ and $A \beta 41$ accumulated in the leptomeningeal blood vessels in the aged SP O subject brains as punctate (Fig. 3C). Furthermore, A 338 and A $\beta 41$ were also detected in CAA in the brains of all cases having SP. Among the cases which had SP analyzed here, $A \beta 40$ and $A \beta 42$ were deposited in both SP and CAAs (Additional file 1: Table S1). In contrast to the $A \beta 40$ - and $A \beta 42$-specific antibodies, the antibodies specific for $A \beta 38$ and $A \beta 41$ labeled only vessels regardless of the pathology. Therefore, it is presumed that $A \beta 38$ and $A \beta 41$ accumulate in vessels before the formation of the SP. Although we cannot confirm whether $\mathrm{A} \beta 38$ and $\mathrm{A} \beta 41$ positive vessels develop CAA, these $A \beta$ species may be generated and accumulate into cerebral vessel walls in pre-pathological conditions.

\section{Discussion}

Here we report a detailed study of $A \beta$ distribution and its isoforms in brains with $\mathrm{AD}, \mathrm{CAA}$, and age-matched controls using MALDI-IMS and IHC. The current technical
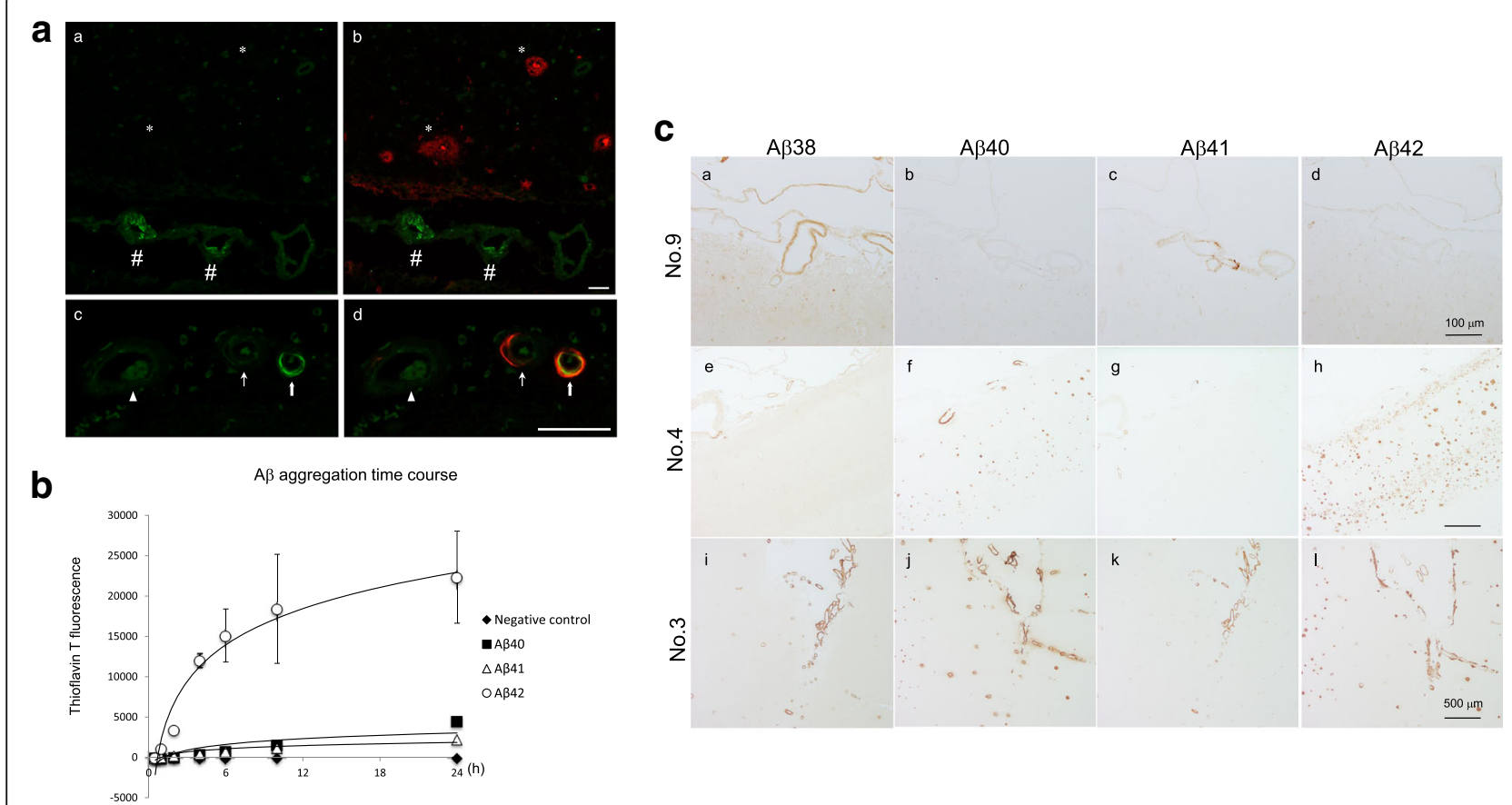

Fig. 3 A: $A \beta 41$ is deposited in leptomeningeal blood vessels. Frozen sections from an AD brain were subjected to the immunostaining using antibodies against $A \beta 41$ (green in $\mathbf{a}-\mathbf{d}$ ), $A \beta 42$ (red in $\mathbf{b}$ ), and $A \beta 40$ (red in $\mathbf{d}$ ). Double immunostaining against both $A \beta 41$ and $A \beta 42$ demonstrated that the anti-A $\beta 41$ antibody labeled the arterioles $(\#)$ in the subarachnoid space, but not the senile plaques $\left(^{*}\right)$ in the parenchyma (a and $\mathbf{b}$ ). Double staining against $A \beta 41$ and $A \beta 40$ is shown. Three different stages of amyloid angiopathies, with weak or no $A \beta 41$ deposition (arrowhead), modest $A \beta 41$ deposition (thin arrow), and with severe $A \beta 41$ deposition (thick arrow) are shown (c and $\mathbf{d}$ ). $A \beta 41$ was found in the amyloid angiopathy with severe $A \beta 40$ deposition. Contrary to the $A \beta 40$, which deposited in the periphery of adventitia, $A \beta 41$ seemed to be localized in the smooth muscles layer of blood vessels. Scale bars $=50 \mu \mathrm{m}$. B: Time course of in vitro $A \beta$ aggregation. Each synthetic $A \beta$ incubated and measured thioflavin T fluorescence. $A \beta 1-42$ aggregates immediately compared to the other variants. $A \beta 1-40$ and $A \beta 1-41$ were similar and showed little aggregation characteristic for $24 \mathrm{~h}$. C: $1 \mathrm{HC}$ for $A \beta$ s accumulation in the occipital cortex sections. $A \beta 38$ and $A \beta 41$ deposited in the leptomeningeal blood vessels in aged SP free brain (NO.9) (a, c). A Ss38, 40, and 41 also deposited in AD (NO.4) and CAA brains (NO.3), but A 440 had little deposits in the cortex, including arterioles $(\mathbf{e}-\mathbf{g}, \mathbf{i}-\mathbf{k})$. Amount of A 42 deposited in subpial granular cell layers and cortex $(\mathbf{h}, \mathbf{l})$. Scale bars $=100 \mu \mathrm{m}(\mathbf{a}-\mathbf{d})$ and $500 \mu \mathrm{m}(\mathbf{e}-\mathbf{l})$ 
advantages of MALDI-IMS allowed us to unveil the distribution of various $A \beta$ species within the same sections of human autopsied brains without specific probes. Furthermore, high resolution $(20 \mu \mathrm{m})$ imaging of the brain of a subject with $\mathrm{AD}$ and severe $\mathrm{CAA}$ clearly demonstrates that $A \beta 1-36$ to $A \beta 1-41$ deposit into leptomeningeal vessel walls, while $A \beta 1-42$ and $A \beta 1-43$ aggregate in the cerebral parenchyma as SP. It is worth noting that MALDI-IMS detected $\mathrm{A} \beta$ deposition as an even dot-like pattern in normal control brains (Additional file 1: Figure S1). Considering that characterization of deposited $\mathrm{A} \beta$ by IMS must be in good agreement with IHC, both IMS and IHC were used and equally contributed to distinguishing $A \beta$ deposits by their location, protein contents, and their morphology.

Multiple pathways of $\beta$-carboxyl-terminal fragment ( $\beta$ CTF) processing by stepwise $\gamma$-secretase cleavage have been previously proposed $[11,16,29]$. In this model, A $\beta 1-41$ is thought to exist in human AD brains, albeit as a minority. In the current study, we have succeeded in detecting the existence of $A \beta 1-41$ in human brains by IMS and $\mathrm{IHC}$ for the first time. According to the $\mathrm{A} \beta$ processing model, $A \beta 1-38$ is derived from $A \beta 1-45$ via $A \beta 1-42$, while $\mathrm{A} \beta 1-41$ is derived from $A \beta 1-45$ by $\gamma$-secretase stepwise cleavage $[11,16,29]$. Of note, $\gamma$-secretase generates easily modifiable full-length $A \beta 1-36$ to $A \beta 1-41$ in the cerebral parenchyma and interstitial fluid (ISF) as a physiological step. Considering that $\gamma$-secretase activity is modulated in AD brains [8], and $A \beta 38$ and $A \beta 41$ have been detected in aged control brains (Fig. 3A, Additional file 1: Table S1), both modulation of $\gamma$-secretase activity and failure of $A \beta$ drainage could be at cause for $A \beta$ accumulation/deposition and CAA in AD brains [33].

An important finding of this study was that $A \beta 41$ was associated with the smooth muscle of arteries, whereas $A \beta 40$ was mostly in the adventitia. It is worth noting that $A \beta 41$ appears to be constrained within the intramural periarterial drainage pathways whereas $\mathrm{A} \beta 40$ appears to have travelled radially across from the smooth muscle basement membranes to the pial-glial basement membranes (Fig. 3C, Additional file 1: Table S1). Previous studies showed that $\mathrm{A} \beta$ is eliminated from ISF through vascular basement membrane to lymph node and carotid artery in the neck, this system is known as the glymphatic system [14, 30, 32]. The $A \beta$ elimination process is forced along the basement membrane of arterial walls by pulse wave [20], which is thought to be slowed in AD brains with aging [5, 31].

MALDI-IMS can individually track the whole distribution of complex molecules having multiple modifications, an advantage over conventional IHC. It is well known that $\mathrm{N}$ terminal truncated $\mathrm{A} \beta$ peptides are abundant in brains of patients diagnosed with sporadic and familial AD [15, 24]. $\mathrm{N} 3 \mathrm{pE}-\mathrm{A} \beta 42$, more so than $\mathrm{A} \beta 1-42$, has been found as a major component of SP $[4,18]$. However, antibodies against the N-terminus of N3pE-A $\beta$ s cannot distinguish both N3pE-
$A \beta 40$ and N3pE-A 342 . Together with the views of a clear contrast of the distribution dependent on $\mathrm{C}$-terminal truncation, it is plausible that the structures of its $\mathrm{C}$-terminal end predefine the dynamics of $\mathrm{A} \beta \mathrm{s}$ rather than those of the $\mathrm{N}$ terminal end. It must be noted that the N3pE-A $\beta 42$ may particularly behave independently from $A \beta 1-42$. Therefore, abundance of $\mathrm{N}$-terminal truncated $\mathrm{A} \beta \mathrm{x}-42(\mathrm{x}=2, \mathrm{~N} 3 \mathrm{pE}, 4$, $5,6,7,8,9,10$, and N11pE) and A $\beta 1-42$ could form the aggregate core in SP. This explains how $\mathrm{C}$-terminally truncated forms of $\mathrm{A} \beta \mathrm{s}$ are more soluble and likely become entrapped more distally in their drainage pathway. In contrast, the more fibrillogenic forms of $A \beta s$ are more prone to aggregation in the extracellular spaces of the brain and may not reach the drainage pathways, particularly in APOE4 positive individuals rather than APOE2/3 [13, 19, 28, 34].

\section{Conclusions}

MALDI-IMS characterized a broad range of $A \beta$ species deposits in brains with AD and CAA. Distinct depositions of N3pE-A $\beta 40$ and N3pE-A $\beta 42$ were comparatively visualized with $A \beta 40$ and $A \beta 42$. $A \beta 1-41$ was first identified in human brains with MALDI-IMS and was confirmed by IHC. The deposition profile of A $\beta 1-41$ drastically differs from $A \beta 1-42$ in its aggregation ability. Thus, $\mathrm{C}$-terminus of $A \beta$ structure determines the deposition /accumulation location in AD brains.

\section{Additional file}

Additional file 1: Figure S1. MALDI-IMS for SP free control subjects. Figure S2. IHC for $A \beta 40(B A 27)$ or $A \beta 42(B C 05)$ in brain with $A D$ and CAA. Figure S3. MALDI-IMS of AD brain (No.3) merged on optic density figure. Figure S4. High resolution $(20 \mu \mathrm{m})$ figure of MALDI-IMS for various C-terminal truncated $A \beta$ in $A D$ with severe CAA.Figure S5. MALDI-IMS for various $\mathrm{N}$-terminal truncated and modified $\mathrm{A} \beta \mathrm{s}$ in $\mathrm{AD}$ with moderate CAA. Figure S6. MALDI-IMS for various N-terminal truncated and modified $A \beta S$ in $A D$ with severe CAA. Figure S7. Anti-AB41 antibody characterization. Supplementary Table S1. (PDF 1494 kb)

\section{Acknowledgements}

We sincerely appreciate the scientific discussions and experimental help of Dr. Satoru Funamoto. This work was supported in part by the Grant-in-Aid for Scientific Research on Innovative Areas (Brain Protein Aging and Dementia Control 26117004; M.I., T.M.). This research was partially supported by the Strategic Research Program for Brain Sciences from Japan Agency for Medical Research and Development, AMED. All experiments were conducted in compliance with the ARRIVE guidelines.

\section{Authors' contributions}

NK, TM, and MI designed the study; NK, SWK and TM performed immunohistochemistry; NI and TN performed the MALDI-IMS; JTF and SM performed the pathological analysis; NK, TM, MI, and YI wrote the manuscript. All authors read and approved the final manuscript.

Ethics approval and consent to participate

This study was approved by the ethics committee at Doshisha University and Tokyo Metropolitan Geriatric Hospital.

Competing interests

The authors declare that they have no competing interests. 


\section{Publisher's Note}

Springer Nature remains neutral with regard to jurisdictional claims in published maps and institutional affiliations.

\section{Author details \\ ${ }^{1}$ Genomics, Proteomics and Biomedical Functions, Department of Life and Medical Systems, Faculty of Life and Medical Sciences, Doshisha University, Kyoto, Japan. ${ }^{2}$ Neuropathology, Department of Life and Medical Systems, Faculty of Life and Medical Sciences, Doshisha University, Kyoto, Japan. ${ }^{3}$ Bruker Daltonics K.K, Yokohama, Japan. ${ }^{4}$ Neuropathology, The Brain Bank for Aging Research, Tokyo Metropolitan Geriatric Hospital and Institute of Gerontology, Tokyo, Japan. ${ }^{5}$ Graduate School of Brain Science, Doshisha University, Kyoto, Japan.}

\section{Received: 19 August 2017 Accepted: 2 October 2017} Published online: 16 October 2017

\section{References}

1. Bateman RJ, Xiong C, Benzinger TL, Fagan AM, Goate A, Fox NC et al (2012) Dominantly inherited Alzheimer network. Clinical and biomarker changes in dominantly inherited Alzheimer's disease. N Engl J Med 367:795-804

2. Braak H, Alafuzoff I, Arzberger T, Kretzschmar H, Del Tredici K (2006) Staging of Alzheimer disease-associated neurofibrillary pathology using paraffin sections and immunocytochemistry. Acta Neuropathol 112:389-404

3. Braak H, Braak E (1991) Neuropathological stageing of Alzheimer-related changes. Acta Neuropathol 82:239-259 Review

4. Harigaya Y, Saido TC, Eckman CB, Prada CM, Shoji M et al (2000) Amyloid beta protein starting pyroglutamate at position 3 is a major component of the amyloid deposits in the Alzheimer's disease brain. Biochem Biophys Res Commun 276:422-427

5. Hawkes CA, Härtig W, Kacza J, Schliebs R, Weller RO, Nicoll JA et al (2011) Perivascular drainage of solutes is impaired in the ageing mouse brain and in the presence of cerebral amyloid angiopathy. Acta Neuropathol 121:431-443

6. Imatsubo T, Odaka A, Suzuki N, Mizusawa H, Nukina N, Ihara Y (1994) Visualization of A beta 42(43) and A beta 40 in senile plaques with endspecific $\mathrm{A}$ beta monoclonals: evidence that an initially deposited species is Abeta 42(43). Neuron 13:45-53

7. Kakuda N, Funamoto S, Yagishita S, Takami M, Osawa S, Dohmae N et al (2006) Equimolar production of amyloid beta-protein and amyloid precursor protein intracellular domain from beta-carboxyl-terminal fragment by gamma-secretase. J Biol Chem 281:14776-14786

8. Kakuda N, Shoji M, Arai H, Furukawa K, Ikeuchi T, Akazawa K et al (2012) Altered $\gamma$-secretase activity in mild cognitive impairment and Alzheimer's disease. EMBO Mol Med 4:344-352

9. Kelley AR, Perry G, Castellani RJ, Bach SB (2016) Laser-induced in-source decay applied to the determination of Amyloid-Beta in Alzheimer's brains. ACS Chem Neurosci 7:261-268

10. Kovacs GG (2016) Molecular Pathological Classification of Neurodegenerative Diseases: Turning towards Precision Medicine. Int J Mol Sci 17:E189. doi: 10.3390/ijms17020189

11. Matsumura N, Takami M, Okochi M, Wada-Kakuda S, Fujiwara H, Tagami S et al (2014) $y$-Secretase associated with lipid rafts: multiple interactive pathways in the stepwise processing of $\beta$-carboxyl-terminal fragment. J Biol Chem 289:5109-5121

12. Miyasaka T, Watanabe A, Saito Y, Murayama S, Mann DM, Yamazaki M et al (2005) Visualization of newly deposited tau in neurofibrillary tangles and neuropil threads. J Neuropathol Exp Neurol 64:665-674

13. Morishima-Kawashima M, Oshima N, Ogata H, Yamaguchi $H_{\text {, Yoshimura M, }}$ Sugihara S et al (2000) Effect of apolipoprotein E allele epsilon4 on the initial phase of amyloid beta-protein accumulation in the human brain. Am J Pathol 157:2093-2099

14. Morris AW, Sharp MM, Albargothy NJ, Fernandes R, Hawkes CA, Verma A et al (2016) Vascular basement membranes as pathways for the passage of fluid into and out of the brain. Acta Neuropathol 131:725-736

15. Portelius E, Bogdanovic N, Gustavsson MK, Volkmann I, Brinkmalm G, Zetterberg $\mathrm{H}$ et al (2010) Mass spectrometric characterization of brain amyloid beta isoform signatures in familial and sporadic Alzheimer's disease. Acta Neuropathol 120:185-193

16. Qi-Takahara Y, Morishima-Kawashima M, Tanimura Y, Dolios G, Hirotani N, Horikoshi Y et al (2005) Longer forms of amyloid beta protein: implications for the mechanism of intramembrane cleavage by gamma-secretase. J Neurosci 25:436-445

17. Reinert J, Richard BC, Klafki HW, Friedrich B, Bayer TA, Wiltfang J et al (2016) Deposition of C-terminally truncated $A B$ species $A B 37$ and $A B 39$ in Alzheimer's disease and transgenic mouse models. Acta Neuropathol Commun 4:24. doi: 10.1186/s40478-016-0294-7.

18. Saido TC, Iwatsubo T, Mann DM, Shimada H, Ihara Y, Kawashima S (1995) Dominant and differential deposition of distinct beta-amyloid peptide species, a beta N3(pE), in senile plaques. Neuron 14:457-466

19. Saito T, Suemoto T, Brouwers N, Sleegers K, Funamoto S, Mihira N et al (2011) Potent amyloidogenicity and pathogenicity of Aß43. Nat Neurosci 14:1023-1032

20. Schley D, Carare-Nnadi R, Please CP, Perry VH, Weller RO (2006) Mechanisms to explain the reverse perivascular transport of solutes out of the brain. J Theor Biol 238:962-974

21. Seeley EH, Caprioli RM (2008) Molecular imaging of proteins in tissues by mass spectrometry. Proc Natl Acad Sci U S A 105:18126-18131

22. Selkoe DJ (2001) Alzheimer's disease: genes, proteins, and therapy. Physiol Rev 81:741-766

23. Selkoe DJ, Hardy J (2016) The amyloid hypothesis of Alzheimer's disease at 25 years. EMBO Mol Med 8:595-608

24. Sergeant N, Bombois S, Ghestem A, Drobecq H, Kostanjevecki V, Missiaen C et al (2003) Truncated beta-amyloid peptide species in pre-clinical Alzheimer's disease as new targets for the vaccination approach. J Neurochem 85:1581-1591

25. Stoeckli M, Knochenmuss R, McCombie G, Mueller D, Rohner T, Staab D et al (2006) MALDI MS imaging of amyloid. Methods Enzymol 412:94-106

26. Stoeckli M, Staab D, Staufenbiel M, Wiederhold KH, Signor L (2002) Molecular imaging of amyloid beta peptides in mouse brain sections using mass spectrometry. Anal Biochem 311:33-39

27. Suzuki N, Iwatsubo T, Odaka A, Ishibashi Y, Kitada C, Ihara Y (1994) High tissue content of soluble beta 1-40 is linked to cerebral amyloid angiopathy. Am J Pathol 145:452-460

28. Tai LM, Bilousova T, Jungbauer L, Roeske SK, Youmans KL, Yu C et al (2013) Levels of soluble apolipoprotein E/amyloid- $\beta$ (AB) complex are reduced and oligomeric $A \beta$ increased with APOE4 and Alzheimer disease in a transgenic mouse model and human samples. J Biol Chem 288:5914-5926

29. Takami M, Nagashima Y, Sano Y, Ishihara S, Morishima-Kawashima M, Funamoto $S$ et al (2009) $\gamma$-Secretase: successive tripeptide and tetrapeptide release from the transmembrane domain of $\beta$-carboxyl terminal fragment. J Neurosci 29:13042-13052

30. Vinters HV (1987) Cerebral amyloid angiopathy. A critical review. Stroke 18: $311-324$

31. Weller RO, Boche D, Nicoll JA (2009) Microvasculature changes and cerebral amyloid angiopathy in Alzheimer's disease and their potential impact on therapy. Acta Neuropathol 118:87-102

32. Weller RO, Djuanda E, Yow HY, Carare RO (2009) Lymphatic drainage of the brain and the pathophysiology of neurological disease. Acta Neuropathol 117:1-14

33. Weller RO, Subash M, Preston SD, Mazanti I, Carare RO (2008) Perivascular drainage of amyloid-beta peptides from the brain and its failure in cerebral amyloid angiopathy and Alzheimer's disease. Brain Pathol 18:253-266

34. Zekonyte J, Sakai K, Nicoll JA, Weller RO, Carare RO (2016) Quantification of molecular interactions between ApoE, amyloid-beta $(A B)$ and laminin: relevance to accumulation of $A \beta$ in Alzheimer's disease. Biochim Biophys Acta 1862:1047-1053

\section{Submit your next manuscript to BioMed Central and we will help you at every step:}

- We accept pre-submission inquiries

- Our selector tool helps you to find the most relevant journal

- We provide round the clock customer support

- Convenient online submission

- Thorough peer review

- Inclusion in PubMed and all major indexing services

- Maximum visibility for your research

Submit your manuscript at www.biomedcentral.com/submit 